\title{
On Huygens' principle for Dirac operators associated to electromagnetic fields
}

\author{
FABIO A.C.C. CHALUB* \\ IMPA, Estrada Dona Castorina, 110 - 22460-320 Rio de Janeiro, RJ, Brazil \\ Manuscript received on July 20, 2001; accepted for publication on August 22, 2001; \\ presented by MANFREDO DO CARMO
}

\begin{abstract}
We study the behavior of massless Dirac particles, i.e., solutions of the Dirac equation with $m=0$ in the presence of an electromagnetic field. Our main result (Theorem 1) is that for purely real or imaginary fields any Huygens type (in Hadamard's sense) Dirac operators is equivalent to the free Dirac operator, equivalence given by changes of variables and multiplication (right and left) by nonzero functions.
\end{abstract}

Key words: Huygens' principle, Dirac operators, electromagnetic fields, Hadamard's problem.

\section{INTRODUCTION}

By 1690 two different theories were disputing the nature of light: for Newton (Newton, 1979), light was composed of particles propagating along straight lines; whereas Huygens believed that light was a mechanical wave propagating in the æther, the hypothetical medium that fulfilled all the space (Shapiro 1980).

Huygens' theory was particularly useful to explain diffraction (" the strange refraction of the Iceland crystal'"), but had some remarkable difficulties to explain the apparent propagation of the light along straight lines. To overcome this difficulty, he wrote his masterpiece "Treatise on Light" (Huygens 1920) where he formulated the principle that bears his name.

In more modern terms (in a formulation essentially due to Fresnel (Fresnel 1816) - for a historical overview, see (Born and Wolf 1980)) we say that each point in the wave front acts as a secondary source of wave and all these waves interfere to produce the new wave front. See Figure 1.

Despite the large use of the term "Huygens' principle"' in the physical community, its precise mathematical formulation is seldom made clear. In 1923, the French mathematician J. Hadamard,

\footnotetext{
*Present address:

Dept. of Mathematics, University of Vienna

Boltzmangasse 9 - A-1090, Wien, Austria

E-mail: fabio@impa.br
} 

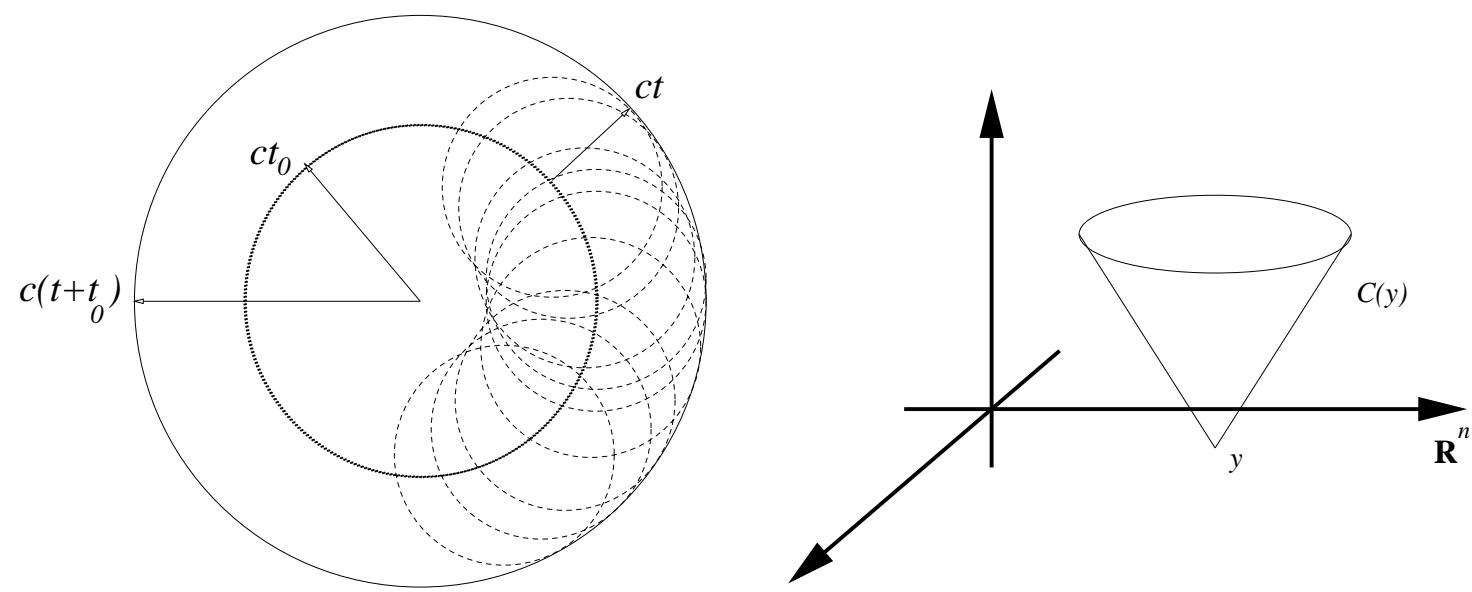

Fig. 1 - Huygens' construction of a wave front from the previous one (left). Forward light cone with vertex in $y$ (right).

in a series of lectures at Yale University, formulated in mathematical terms three different meanings of "Huygens' principle"' he found in the literature of his time (Hadamard 1953). This work is concerned with the second definition, called "Hadamard's minor premise."

We define the forward light cone with vertex in $y$ as

$$
\mathcal{C}^{+}(y)=\left\{x \in \mathbb{R}^{n+1} \mid\left(x^{0}-y^{0}\right)^{2}-\sum_{i=1}^{n}\left(x^{i}-y^{i}\right)^{2}=0, x^{0}>y^{0}\right\} .
$$

and the fundamental solution (also known as Green's function) $\phi$ of an operator $\mathcal{L}$

$$
\mathcal{L} \phi=\delta_{y},
$$

where $\delta_{y}$ is the Dirac-delta distribution supported in $y$.

We say that $\mathcal{L}$ (and, by extension, its fundamental solution $\phi$ ) possesses Huygens' property (or obeys Huygens' principle) if, for every $y, \phi$ is supported in the forward light cone with vertex in $y$.

This definition is called ' Hadamard's minor premise.' See Figure 1.

As Huygens' principle was originally formulated for light propagation, a physical phenomenon modeled by the wave equation, it is useful to discuss Huygens' principle in Hadamard's minor premise sense in this case first.

The wave equation is a partial differential equation in $n+1$ variables, $\left(x^{0}, x^{1}, \cdots, x^{n}\right)$, where $x^{0}$ is taken as time, and the other variables are the space variables. Explicitly, it is an equation for a function $u$ such that

$$
\square u \stackrel{\text { def }}{=} \frac{\partial^{2} u}{\left(\partial x^{0}\right)^{2}}-\sum_{i=1}^{n} \frac{\partial^{2} u}{\left(\partial x^{i}\right)^{2}}=0 .
$$

The above equation is known as the wave equation in $n$ space variables. With these definitions we take the velocity of propagation $c$ equals 1 . If we do not want to fix the value of $c$, we change in the above and the following expression the value of $x^{0}$ by $c x^{0}$. 
It is a well known fact that the wave operator possesses Huygens' principle for odd $n$ greater than or equal to 3 and does not posses it otherwise. See (Folland 1997).

Hadamard's problem consists in classifying all second order hyperbolic operators which obey Huygens' principle, up to trivial relations. The latter consist in successive combinations of changes of variables, and left or right multiplication of the operator by a never-singular smooth function. This problem has not been fully solved, but progress has been made recently. An extensive and up-to-date review can be found in Berest's work (Berest 1998). Previous work on the subject of Huygens' principle for Dirac operators are (Chalub and Zubelli 2000, Chalub and Zubelli 2001a).

In this work we will study the validity of Huygens' principle for Dirac operators in the presence of an electromagnetic field. In Section 2, we briefly introduce Clifford Algebras, that are instrumental to the definition of Dirac Operators. In Section 3, we present the Hadamard expansion and re-define Huygens' principle in terms of its truncation. In Section 4, we develop some comments about the physical nature of Huygens' principle, in particular in the presence of electromagnetic field and prove the main theorem of this article, namely, that every Dirac operator in the presence of a real or purely imaginary field with Huygens' property is trivially equivalent to the free Dirac operator (Theorem 1).

\section{CLIFFORD ALGEBRAS AND DIRAC OPERATORS}

Dirac operators appear in Mathematical Physics in the framework of the construction of a relativistic electron theory. In a celebrated paper, Dirac (Dirac 1928) defined a Lorentz-covariant Hamiltonian (and consequently first-order in space variables, as Schrödinger equation is first order in time) whose square furnishes the Klein-Gordon equation.

Let $g^{\mu \nu}=\operatorname{diag}[1,-1, \cdots,-1]$ denote the Minkowski tensor. Associated to $g^{\mu \nu}$ we can construct a Clifford Algebra. It is an associative algebra (with identity $I$ ) over the reals generated by all linear combinations of the form $\left(\gamma^{0}\right)^{m_{0}}\left(\gamma^{1}\right)^{m_{1}} \cdots\left(\gamma^{n}\right)^{m_{n}}, \quad m_{\mu} \in\{0,1\}$, where the matrices $\left\{\gamma^{\mu}\right\}$, obey the relation $\gamma^{\mu} \gamma^{\nu}+\gamma^{\nu} \gamma^{\mu}=2 g^{\mu \nu} I$. The matrices $\left\{\gamma^{\mu}\right\}$, which are called Dirac matrices, are linearly independent (Gilbert and Murray 1991, Marchuk 1998).

Dirac operators are defined by:

$$
\mathcal{D}=\gamma^{\mu} \partial_{\mu}+v
$$

where the summation for repeated indices is implied. See (Thaller 1992) for an introduction on the subject. We shall adopt the notation $\not \partial=\gamma^{\mu} \partial_{\mu}$ and restrict ourselves to the case where $v$ is of electromagnetic (or vector) form, $v=a_{\mu} \gamma^{\mu}$.

\section{HUYGENS' PRINCIPLE AND HADAMARD EXPANSIONS}

In this section we shall look for fundamental solutions of Dirac operators. A fundamental solution for $\not \partial+v$ is a solution of $(\not \partial+v) \Phi=\delta_{y}$, where $\delta_{y}$ denotes Dirac-delta distribution supported at an arbitrary point $y$ in space-time. 
Let $\lambda(x, y)=\sqrt{\left(x^{\mu}-y^{\mu}\right)\left(x_{\mu}-y_{\mu}\right)}$ denote the Minkowski distance between two points $x$ and $y$.

The first important concept to be recalled is the so-called Riesz kernel. It was introduced by M. Riesz in order to unify the treatment of elliptic and hyperbolic problems. They are given by distributions in $\mathcal{D}^{\prime}\left(\mathbb{R}^{n+1}\right)$ defined first for $\operatorname{Re}(\alpha)$ sufficiently large by

$$
\Lambda^{\alpha}=\left\{\begin{array}{l}
N(\alpha) \lambda^{\alpha}, \text { if }\left(x^{\mu}-y^{\mu}\right)\left(x_{\mu}-y_{\mu}\right) \geq 0 \\
0, \text { otherwise. }
\end{array}\right.
$$

Here, the normalizations constant $N(\alpha)$ is given by

$$
N(\alpha)=\left[2^{\alpha+n} \pi^{(n-1) / 2} \Gamma\left(\frac{\alpha+n+1}{2}\right) \Gamma\left(\frac{\alpha}{2}+1\right)\right]^{-1} .
$$

Then, $\Lambda^{\alpha}$ is extended analytically for all values of $\alpha$ in the complex plane by means of $\square \Lambda^{\alpha}=$ $\Lambda^{\alpha-2}$, which is satisfied for all $\alpha$ with sufficiently large real part. It is not hard to check that $\Lambda^{-n-1}=\delta_{y}$. See (Folland 1997).

We shall say that a fundamental solution $\Phi$ obeys Huygens' principle if supp $\Phi \subset \mathcal{C}^{+}(y) \forall y \in$ $\mathbb{R}^{n+1}$.

Adapting Hadamard's seminal idea from the wave operator case to the one at hand, we look for series expansion for the fundamental solution

$$
\Phi=\sum_{m=0}^{\infty} \not \partial^{-m} \Theta^{-\alpha_{0}} s_{m}=\sum_{m=0}^{\infty}\left\{\Theta^{\alpha_{0}+2 m} s_{2 m}+\Lambda^{\alpha_{0}+2 m} s_{2 m+1}\right\},
$$

where $s_{m}$ is a matrix coefficient, $\alpha_{0} \rightarrow-n+1, \Theta^{\alpha}=\not \partial \Lambda^{\alpha}$, which we call Dirac kernels, and $\Lambda^{\alpha}$ are Riesz kernels. It can be shown that $\Theta^{\alpha}$ satisfies Huygens' property for $\alpha=0,-2,-4, \cdots$; while $\Lambda^{\alpha}$ does it for $\alpha=-2,-4, \cdots$.

If $\Phi$ is a fundamental solution, using the properties of Riesz and Dirac kernels, we have the Hadamard's recursion for the coefficients $s_{m}$

$$
\begin{aligned}
s_{0}+\frac{2}{\alpha_{0}+n-1}\left(x^{\mu}-y^{\mu}\right)\left(\partial_{\mu}+a_{\mu}\right) s_{0} & =1, \alpha_{0} \rightarrow-n+1, \\
s_{2 m+1} & =(\not \partial+v) s_{2 m}, \\
s_{2 m}+\frac{1}{m}\left(x^{\mu}-y^{\mu}\right)\left(\partial_{\mu}+a_{\mu}\right) s_{2 m} & =-(\not \partial+v) s_{2 m-1} .
\end{aligned}
$$

In all these cases $s_{m}$ should be smooth (in particular when $y^{\mu} \rightarrow x^{\mu}$ ) and we impose normalization $s_{0}(y, y)=1$.

We solve equation (1)

$$
s_{0}(x, y)=\exp \left(-\int_{0}^{1}\left(x^{\mu}-y^{\mu}\right) a_{\mu}(y+z(x-y)) d z\right) .
$$


An important property of this series is that it is unique, provided we require $s_{m}$ to be smooth. The uniqueness for (2) is trivial. In the case of (3) it should be proved. Let $s^{1}$ and $s^{2}$ be two different solutions of (3). So, the difference $s^{1}-s^{2}=S_{2 m}$ is smooth and satisfies

$$
S_{2 m}+\frac{1}{m}\left(x^{\mu}-y^{\mu}\right)\left(\partial_{\mu}+a_{\mu}\right) S_{2 m}=0
$$

We find that

$$
S_{2 m}=\tilde{S} \exp \left(-\int_{0}^{1}\left(x^{\mu}-y^{\mu}\right) a_{\mu}(y+z(x-y)) d z\right)
$$

with $\tilde{S}$ satisfying

$$
\tilde{S}+\frac{1}{m}\left(x^{\mu}-y^{\mu}\right) \partial_{\mu} \tilde{S}=0 .
$$

The only smooth solution in the vicinity of the vertex $y$ is given by $\tilde{S}=0$, then $S_{2 m}=0$.

Bearing in mind this fact it is easy to prove the equivalence between Huygens' principle and the truncation of the Hadamard series:

REMARK 1. A Dirac operator in the form $\not \partial+v$ possesses Huygens' property in odd spatial dimension $n \Longleftrightarrow$ its Hadamard series truncates at $n$, i.e, $s_{m}=0$ for $m \geq n$.

Proof. Proving this remark is quite simple. In the $\Leftarrow$ direction, it follows from the properties of Dirac and Riesz kernels. In the other direction, it is a consequence of the uniqueness of the Hadamard expansion.

\section{HUYGENS' PRINCIPLE IN AN ELECTROMAGNETIC FIELD}

In this section we shall consider potentials of the form $v=a_{\mu} \gamma^{\mu}$.

Potentials of the form $a_{\mu} \gamma^{\mu}$ are called electromagnetic potentials because they correspond to including an electromagnetic field in the physical situation under consideration, except for a multiplication by the complex unit $i$. If the physical potential is given by the "four-vector" $A_{\mu}$ the new momentum is given by $i \partial_{\mu}-A_{\mu}$, so the "physical case", in the expression of $v$, is given by purely imaginary components.

If we write the potential as a 1-form $\mathbf{a}=a_{\mu} d x^{\mu}$, the electromagnetic field is given by its exterior derivative

$$
\mathbf{F}=d \mathbf{a}
$$

The 2-form $\mathbf{F}$ is often described by its tensor components,

$$
F_{\mu \nu}=\partial_{\mu} a_{\nu}-\partial_{\nu} a_{\mu}
$$

Electromagnetic fields obey Maxwell's equation, which in vacuum are written

$$
\partial^{\mu} F_{v \mu}=j_{v} \quad \partial_{[\mu} F_{v \rho]}=0
$$


In these equations $j_{v}$ is the density of electrical charge and current, and the notation [.] means that we sum over all the permutation of the indexes in brackets, taking in consideration the parity of the permutation (For example: $B_{[\mu \nu]}=B_{\mu \nu}-B_{\nu \mu}, B_{[\mu} C_{\nu]}=B_{\mu} C_{\nu}-B_{\nu} C_{\mu}$ and $B_{[\mu} C_{\nu \rho]}=$ $B_{\mu} C_{\nu \rho}+B_{\nu} C_{\rho \mu}+B_{\rho} C_{\mu \nu}-B_{\mu} C_{\rho \nu}-B_{\nu} C_{\mu \rho}-B_{\rho} C_{\nu \mu}$.). The second equation is automatically obeyed, as long as $F_{\mu \nu}$ is obtained from a potential. Inside matter the second Maxwell equation remains valid (which implies, from Poincaré's lemma that, locally, the electromagnetic field can be obtained from a potential).

In classical physics the field at each point is observable, while the potentials are considered just a technique for calculating such field, having no separate reality (This changes dramatically in Quantum Mechanics, as can be seen by the Aharonov-Bohm effect. See, for example (Sakurai 1967)). This means that adding an exact form $d \theta$ to the potential should not change the field (which is obvious from the fact that $d^{2}=0$ ). In terms of its components, this means to change from $a_{\mu}$ to $a_{\mu}^{\prime}=a_{\mu}+\partial_{\mu} \theta$. As the Huygens' property is a physically sound problem, the validity or not of the Huygens' principle cannot depend on the particular choice of the electromagnetic gauge (to understand the idea that Huygens' principle is physically observable we should understand the meaning of $\Psi$, the solution of Dirac equation. Following Section 1.7.3 of (Thaller 1992), we say that for every Borel-measurable set $\mathcal{B} \in \mathbb{R}^{3}$ the probability of finding the particle in $\mathcal{B}$ is given by $\langle\Psi, E(\mathcal{B}) \Psi\rangle$, where $E(\mathcal{B})$ is a projection operator in $\mathcal{B}$ and the inner product should be interpreted as Hilbert space inner product where the Dirac operator is acting and the solution $\Psi$ lives. In simpler words, we have a non-zero probability of finding the particle only on the support of the wave-function.).

The change of the gauge is a particular kind of trivial transformation, as defined in the introduction. We see immediately

$$
\not \partial+\left(a_{\mu}+\partial_{\mu} \theta\right) \gamma^{\mu}=e^{-\theta}\left(\not \partial+a_{\mu} \gamma^{\mu}\right) e^{\theta},
$$

so the change of the gauge does not change the validity of the Huygens' principle.

Thus, the invariance of Huygens' principle by change of gauge can be concluded by two distinct reasonings, coming from two different points of view, historically unrelated. We point out that, as far as we know, the fact that the change of electromagnetic gauge is a particular case of trivial transformations as defined by Hadamard was never mentioned in the literature.

After these remarks we are ready to state:

THEOREM 1. If a Dirac operator $\not \partial+a_{\mu} \gamma^{\mu}$ obeys Huygens' property for real or purely imaginary potentials $a_{\mu}$ in $3+1$ dimensions, then it is equivalent to the free operator $\not \partial$.

Proof. Let us define

$$
f_{\mu}(x, y) \stackrel{\text { def }}{=} a_{\mu}(x)-\partial_{\mu} \int_{0}^{1}\left(x^{\nu}-y^{v}\right) a_{v}(\xi(z)) d z,
$$

where $\xi(z)=y+z(x-y)$. The first remark is that

$$
\partial_{\mu} f_{v}-\partial_{v} f_{\mu}=\partial_{\mu} a_{v}-\partial_{v} a_{\mu},
$$


which shows that $\mathbf{f}$ and a are gauge equivalent. Explicitly, the change of gauge is given by

$$
\not \partial+f_{\mu} \gamma^{\mu}=e^{-\theta}\left(\not \partial+a_{\mu} \gamma^{\mu}\right) e^{\theta}
$$

where

$$
\theta(x, y) \stackrel{\text { def }}{=}-\int_{0}^{1}\left(x^{\mu}-y^{\mu}\right) a_{\mu}(\xi(z)) d z .
$$

From now on, the calculations will be made in the new gauge.

The functions $f_{\mu}$ can be re-written as

$$
f_{\mu}(x, y)=\int_{0}^{1}\left(x^{\nu}-y^{v}\right) F_{\nu \mu}(\xi(z)) z d z,
$$

and, so, by the anti-symmetry of the tensor $F_{\mu \nu}$ we have

$$
\left(x^{\mu}-y^{\mu}\right) f_{\mu}(x, y)=0 \quad \forall x, y \in \mathbb{R}^{4} .
$$

Since Hadamard's recursion stops at the second term, Hadamard's recursion becomes

$$
\begin{aligned}
s_{0}+\frac{2}{\alpha_{0}+n-1}\left(x^{\mu}-y^{\mu}\right) \partial_{\mu} s_{0} & =1, \alpha_{0} \rightarrow-n+1, \\
s_{1}=\left(\not \partial+f_{\mu} \gamma^{\mu}\right) 1 & =\gamma^{\mu} f_{\mu} \stackrel{\text { def }}{=} f . \\
s_{2}+\left(x^{\mu}-y^{\mu}\right) \partial_{\mu} s_{2} & =-(\not \partial+f) f, \\
(\not \partial+f) s_{2} & =0 .
\end{aligned}
$$

The first equation can be easily solved to get $s_{0}=1$.

We shall study compatibility conditions between the last two equations. We start applying Dirac operator to the first:

$$
2 \not \partial u_{2}+\left(x^{\mu}-y^{\mu}\right) \partial_{\mu} \not \partial u_{2}=-\not \partial(\not \partial+f) f .
$$

With the second condition this can be written

$$
2 f u_{2}+\left(x^{\mu}-y^{\mu}\right)\left(\partial_{\mu} f\right) u_{2}+f\left(x^{\mu}-y^{\mu}\right) \partial_{\mu} u_{2}=\not \partial(\not \partial+f) f .
$$

Applying $\partial_{\nu}$ to equation (5) we find that $\left(x^{\mu}-y^{\mu}\right) \partial_{\nu} f_{\mu}=-f_{\nu}$ which allows one to write the equation

$$
\left(x^{\mu}-y^{\mu}\right) \partial_{\mu} f=\gamma^{\mu}\left(x^{\nu}-y^{\nu}\right) F_{\nu \mu}-f .
$$

Finally we write

$$
\gamma^{\mu}\left(x^{\nu}-y^{\nu}\right) F_{\nu \mu} u_{2}=(\not \partial+f)(\not \partial+f) f .
$$

The RHS of last equation is

$$
(\not \partial+f)(\not \partial+f) f=\square f+\not \partial f^{2}+f \not \partial f+f^{3} .
$$


For $x=y$, it is immediate to see that $f(x, y)=0$. So, the only non-zero term in $x=y$ in the RHS is $\square f$. Actually,

$$
\square f_{\mu}=\partial^{\rho} \partial_{\rho} \int_{0}^{1}\left(x^{\nu}-y^{\nu}\right) F_{\nu \mu}(\xi) z d z=2 \int_{0}^{1} \partial^{\rho} F_{\rho \mu}(\xi) z^{2} d z+\int_{0}^{1} \square F_{v \mu}(\xi) z^{3} d z .
$$

Letting $y \rightarrow x$

$$
\square f(x, x)=\frac{2}{3} \gamma^{\mu} \partial^{v} F_{v \mu}(x) .
$$

As the LHS of equation ( 7$)$ is zero at $(x, x)$, we conclude that

$$
\partial^{v} F_{\mu \nu}=0 \quad \mu=0, \cdots, n
$$

is a necessary condition for the operator $\not \partial+a_{\mu} \gamma^{\mu}$ to be a Huygens one. This can be interpreted as the absence of sources.

To get a second necessary condition, we apply

$$
\tilde{\partial}_{\eta} \stackrel{\text { def }}{=} \frac{\partial}{\partial y^{\eta}}
$$

to the LHS of equation (7) and evaluate it at $(x, x)$. We get

$$
\begin{aligned}
\tilde{\partial}_{\eta} \square f(x, x) & =\frac{1}{6} \gamma^{\mu} \partial_{\eta} \partial^{\rho} F_{\rho \mu}-\frac{1}{4} \gamma^{\mu} \square F_{\eta \mu} ; \\
\tilde{\partial}_{\eta} \not \partial f(x, x)^{2} & =2 \gamma^{\mu}\left(\partial_{\mu} f_{\nu}\right)(x, x)\left(\tilde{\partial}_{\eta} f^{v}\right)(x, x) ; \\
\tilde{\partial}_{\eta}(f \not \partial f)(x, x) & =\left(\tilde{\partial}_{\eta} f\right)(x, x)(\not \partial f)(x, x) ; \\
\tilde{\partial}_{\eta} f(x, x)^{3} & =0 .
\end{aligned}
$$

Taking into account equations (4) and (8) the above system can be re-written as

$$
\tilde{\partial}_{\eta}(\not \partial+f)^{2} f(x, x)=\frac{1}{2} \gamma^{\mu}\left(F^{2}\right)_{\mu \nu}-\frac{1}{4} \gamma^{\mu} \gamma^{\nu} \gamma^{\rho} F_{\eta \mu} F_{\nu \rho}
$$

where $\left(F^{2}\right)_{\mu \nu}$ indicates the $(\mu, v)$ component of the tensor given by the square of the matrix $F$, $\left(F^{2}\right)_{\mu \nu} \stackrel{\text { def }}{=} F_{\mu \rho} F_{\nu}^{\rho}$.

The RHS of equation (7) gives

$$
\tilde{\partial}_{\eta}\left[\gamma^{\mu}\left(x^{\nu}-y^{\nu}\right) F_{\nu \mu} u_{2}\right](x, x)=-\gamma^{\mu} F_{\eta \mu} u_{2}(x, x),
$$

and $u_{2}(x, x)$ can be easily obtained from equation (6):

$$
u_{2}(x, x)=-(\not \partial+f) f(x, x)=\frac{1}{2} \gamma^{\mu} \gamma^{v} F_{\mu \nu} .
$$

We gather all this information in a single equation, written in the Clifford base as

$$
\gamma^{\mu}\left(F^{2}\right)_{\mu \eta}+\frac{3}{4} \sum_{\mu<\nu<\rho} \gamma^{\mu} \gamma^{v} \gamma^{\rho} F_{\eta[\mu} F_{\nu \rho]}=0
$$


where, as before [·] means that we sum over all the permutation of $\mu, v$ and $\rho$ taking in consideration the parity of the permutation.

From the independence of the generators of the Clifford Algebra, we conclude that

$$
\left(F^{2}\right)_{\mu \nu}=0 \quad \text { and } \quad F_{\eta[\mu} F_{\nu \rho]}=0 \quad \forall \mu, v, \rho, \eta=0, \cdots, 3
$$

Let us write $\left(F^{2}\right)$ componentwise:

$$
\begin{gathered}
\left(F^{2}\right)_{\mu \nu}=F_{\mu \rho} F_{\nu}^{\rho}= \\
=\left(\begin{array}{cccc}
E_{x}^{2}+E_{y}^{2}+E_{z}^{2} & E_{z} B_{y}-E_{y} B_{z} & E_{x} B_{z}-E_{z} B_{x} & E_{y} B_{x}-E_{x} B_{y} \\
E_{z} B_{y}-E_{y} B_{z} & B_{y}^{2}+B_{z}^{2}-E_{x}^{2} & -E_{x} E_{y}-B_{x} B_{y} & -E_{x} E_{z}-B_{x} B_{z} \\
E_{x} B_{z}-E_{z} B_{x} & -E_{x} E_{y}-B_{x} B_{y} & B_{x}^{2}+B_{z}^{2}-E_{y}^{2} & -E_{y} E_{z}-B_{y} B_{z} \\
E_{y} B_{x}-E_{x} B_{y} & -E_{x} E_{z}-B_{x} B_{z} & -E_{y} E_{z}-B_{y} B_{z} & B_{x}^{2}+B_{y}-E_{z}^{2}
\end{array}\right),
\end{gathered}
$$

where $E_{x}, E_{y}, E_{z}, B_{x}, B_{y}$ and $B_{z}$ are the $x, y$ and $z$ components of the electric and magnetic fields, respectively.

From the condition $F^{2}=0$ and the hypothesis that all the potentials are either real or purely imaginary (so are the fields), we conclude that $E_{x}=E_{y}=E_{z}=B_{x}=B_{y}=B_{z}=0$, so $F_{\mu \nu}=0$ and $\mathbf{a}$ is gauge equivalent to the zero field.

REMARK 2. If we relax the hypothesis that the potential is real or purely imaginary, then we can find a large class of Huygens potentials. One family of examples is given by

$$
a_{0}=-a_{3}=\int_{\mathbb{R}} \varepsilon(\omega) e^{i \omega\left(x^{3}-x^{0}\right)}\left(x^{1}-i x^{2}\right) d \omega \quad a_{1}=a_{2}=0,
$$

where $\varepsilon(\omega)$ is any function such that $\varepsilon(\omega)=\varepsilon^{*}(-\omega)$ and ${ }^{*}$ denotes complex conjugation.

\section{CONCLUSIONS}

Huygens' principle has been subject of extensive investigation in recent times. For reviews in two different directions, see (Berest 1998) and (Belger et al. 1997). One of its most striking characteristics is its relations to integrability (see (Berest and Veselov 1994)). In the wave operator case, potentials of Huygens' type are, after a suitable change of variables, solutions of the Kortewegde Vries equation

$$
u_{t}=u_{x x x}-6 u u_{x}
$$

For Dirac operators, the relation between Huygens' principle and integrability involves the AKNS (after Ablowitz, Kaup, Newell and Segur) hierarchy, which in turn is an example of integrable matrix systems. This is proved in (Chalub and Zubelli 2001b).

A natural follow up of the present work is to relate Huygens' principle for Dirac operators associated to the electromagnetic field and integrable systems. 


\section{ACKNOWLEDGMENTS}

I thank Prof. Jorge P. Zubelli for introducing me on this subject and for many suggestions. This work was supported by the Brazilian Agencies CNPq and CAPES.

\section{RESUMO}

Neste artigo estudamos o comportamento das soluções da equação de Dirac sem massa ("massless Dirac particles") na presença de um campo eletromagnético. Nosso resultado (Teorema 1) indica que para campos reais ou puramente imaginários todo operador de Dirac que obedece ao princípio de Huygens é equivalente ao operador trivial, equivalência dada por mudanças de variáveis e multiplicação, à direita ou esquerda, por funções não singulares.

Palavras-chave: princípio de Huygens, operadores de Dirac, campos eletromagnéticos, problema de Hadamard.

\section{REFERENCES}

Belger M, Schimming R and Wünsch V. 1997. A survey on Huygens' principle. Z Anal Anwendungen, 16(1): 9-36. Dedicated to the memory of Paul Günther.

Berest Y AND VeSElov AP. 1994. The Huygens principle and integrability. Uspekhi Mat Nauk, 49(6(300)): 7-78.

BEREST Y. 1998. Hierarchies of Huygens' operators and Hadamard's conjecture. Acta Appl Math, 53(2): 125-185.

Born M And Wolf E. 1980. Eletromagnetic Theory of Propagation, Interference and Diffraction of Light. Pergamon Press, sixth edition.

Chalub FACC and Zubelli JP. 2000. Integrable systems, Huygens' principle, and Dirac operators. In Proceedings of the Workshop on Nonlinearity, Integrability and All That: Twenty Years after NEEDS '79 (Gallipoli, 1999), pages 89-96, River Edge, NJ. World Sci. Publishing.

Chalub FACC and Zubelli JP. 2001a. On Huygens' Principle for Dirac Operators and Nonlinear Evolution Equations. J Nonlin Math Phys, 8, Supplement: 62-68.

Chalub FACC and Zubelli JP. 2001b. Huygens' Principle, Dirac Operators and Rational Solutions of the AKNS Hierarchy. Submitted.

Dirac PAM. 1928. The Quantum Theory of the Electron. Proc Roy Soc of London A, 117: 610-624.

Fresnel A. 1816. Ann Chim et Phys, (2), 1, 239. in Oeuvres, Vol 1, 89, 129.

FoLLAND GB. 1997. Fundamental solutions for the wave operator. Exposition Math, 15(1): 25-52.

Gilbert JE And Murray MAM. 1991. Clifford algebras and Dirac operators in harmonic analysis. Cambridge University Press, Cambridge.

Hadamard J. 1953. Lectures on Cauchy's problem in linear partial differential equations. Dover Publica- 
tions, New York.

Huygens C. 1920. Traité de La Lumiére, où sont expliquées les causes de ce qui luy-arrive dans la reflexion, et dans la refraction et particulièrement dans l'etrange refraction du Cristal D'Islande. Societé Hollandaise des Sciences, 1920. Reprint of the 1690 original, Librarie Marchand.

MarchuK NG. 1998. Dirac $\gamma$-equation, classical gauge fields and Clifford algebra. Adv Appl Clifford Algebras, 8(1): 181-225.

Newton I. 1979. Opticks. A treatise of the Reflexion, Inflexion and Colours of Light. Dover Publications, New York.

SaKuRAi JJ. 1967. Advanced Quantum Mechanics. Addison Wesley.

Shapiro A. 1980. Huygens' kinematic theory of light. In Studies on Christiaan Huygens. Swets and Zeitlinger B.V., Lisse.

Thaller B. 1992. The Dirac equation. Springer-Verlag, Berlin. 